\title{
Vocal characteristics of prairie dog alarm calls across an urban noise gradient
}

Shannon, Graeme; McKenna, Megan F.; Wilson-Henjum, Grete; Angeloni, Lisa;

Crooks, Kevin; Wittemyer, George

\section{Behavioral Ecology}

DOI:

10.1093/beheco/arz200

Published: 01/03/2020

Peer reviewed version

Cyswllt i'r cyhoeddiad / Link to publication

Dyfyniad o'r fersiwn a gyhoeddwyd / Citation for published version (APA):

Shannon, G., McKenna, M. F., Wilson-Henjum, G., Angeloni, L., Crooks, K., \& Wittemyer, G. (2020). Vocal characteristics of prairie dog alarm calls across an urban noise gradient. Behavioral Ecology, 31(2), 393-400. https://doi.org/10.1093/beheco/arz200

\section{Hawliau Cyffredinol / General rights}

Copyright and moral rights for the publications made accessible in the public portal are retained by the authors and/or other copyright owners and it is a condition of accessing publications that users recognise and abide by the legal requirements associated with these rights.

- Users may download and print one copy of any publication from the public portal for the purpose of private study or research.

- You may not further distribute the material or use it for any profit-making activity or commercial gain

- You may freely distribute the URL identifying the publication in the public portal ?

Take down policy

If you believe that this document breaches copyright please contact us providing details, and we will remove access to the work immediately and investigate your claim. 
1 Vocal characteristics of prairie dog alarm calls across an urban noise gradient

3 Graeme Shannon ${ }^{1,2}$, Megan F McKenna ${ }^{3}$, Grete E Wilson-Henjum ${ }^{2}$, Lisa M Angeloni ${ }^{4}$,

4 Kevin R. Crooks ${ }^{2}$, George Wittemyer ${ }^{2}$,

$7 \quad{ }^{1}$ School of Natural Sciences, Bangor University, Bangor, United Kingdom

$9 \quad{ }^{2}$ Department of Fish, Wildlife, and Conservation Biology, Colorado State University,

10 Fort Collins, Colorado, United States of America

11

$12{ }^{3}$ National Park Service, Natural Sounds and Night Skies Division, Fort Collins, CO

1380525, USA

14

$15{ }^{4}$ Department of Biology, Colorado State University, Fort Collins, Colorado, United States

16 of America

17

18 Running title: Prairie dog alarm calls in noise

19

20 Correspondence email:

21 g.shannon@bangor.ac.uk 


\section{Abstract}

25 Increasing anthropogenic noise is having a global impact on wildlife, particularly due to

26 the masking of crucial acoustical communication. However, there have been few studies

27 examining the impacts of noise exposure on communication in free-ranging terrestrial

28 mammals. We studied alarm calls of black-tailed prairie dogs (Cynomys ludovicianus)

29 across an urban gradient to explore vocal adjustment relative to different levels of noise

30 exposure. There was no change in the frequency $5 \%$, peak frequency or duration of the

31 alarm calls across the noise gradient. However, the minimum frequency - a commonly

32 used, yet potentially compromised metric - did indeed show a positive relationship with

33 noise exposure. We suspect this is a result of masking of observable call properties by

34 noise, rather than behavioural adjustment. In addition, the proximity of conspecifics and

35 the distance to the perceived threat (observer) did affect the frequency $5 \%$ of alarm calls.

36 These results reveal that prairie dogs do not appear to be adjusting their alarm calls in

37 noisy environments but likely do in relation to their social context and the proximity of a

38 predatory threat. Anthropogenic noise can elicit a range of behavioural and physiological

39 responses across taxa, but elucidating the specific mechanisms driving these responses

40 can be challenging, particularly as these are not necessarily mutually exclusive. Our

41 research sheds light on how prairie dogs appear to respond to noise as a source of

42 increased risk, rather than as a distraction or through acoustical masking as shown in

43 other commonly studied species (e.g. fish, songbirds, marine mammals).

45 Key words: acoustics, mammal, anthropogenic disturbance, communication, masking,

46 predation 


\section{Introduction}

48 Human-induced rapid environmental change is having far-reaching impacts on natural

49 ecosystems across the globe, affecting animal behaviour, demographic processes and

50 community composition (Sih et al. 2011). The pervasive nature of rising anthropogenic

51 noise levels across terrestrial and aquatic habitats provides a prime example of how

52 human activities can dramatically alter the environment over a comparatively short time

53 frame (Barber et al. 2010). During the past two decades, increasing research effort has

54 explored the effects of noise on animal behaviour and demography, with particular focus

55 on how anthropogenic noise affects acoustic communication (Shannon, McKenna, et al.

56 2016).

57 Songbirds in particular have been the focus of numerous studies on the effects of

58 anthropogenic noise on behaviour and communication in urban environments, due to

59 their relative abundance, the important role of vocal communication in many aspects of

60 their behaviour (e.g., territoriality, mate attraction and agonistic social interactions) and

61 the established methodology for studying changes in song structure and singing

62 behaviour (Slabbekoorn 2013). The seminal paper by Slabbekoorn and Peet (2003),

63 which demonstrated that elevated noise levels in urban environments significantly altered

64 vocal communication in great tits (Parus major), was a key catalyst for research effort on

65 this topic. Scientists exploring avian acoustic communication have demonstrated a range

66 of responses to mitigate the effects of noise exposure, which include adjusting the time of

67 vocalising (Fuller et al. 2007), increasing the amplitude of the call (Lowry et al. 2012),

68 lengthening the duration of the call (Díaz et al. 2011), reducing syllable rate (Potvin et al.

69 2011) and shifting the minimum call frequency upwards (Slabbekoorn and Ripmeester 
70 2008). These behavioural adjustments are believed to be adaptive responses that reduce

71 the masking of key signals by low frequency anthropogenic noise, which is

72 predominantly concentrated at $<2 \mathrm{KHz}$ (Brumm et al. 2017). Furthermore, evidence

73 indicates that anthropogenic noise exposure may structure animal communities (Francis

74 et al. 2009; Proppe et al. 2013), as species that vocalise at lower frequencies with limited

75 behavioural flexibility are forced to adjust their distribution (Francis 2015). This suggests

76 responses to noise are likely conditioned on the degree of plasticity in communication

77 modalities.

78 Although the effects of anthropogenic noise on acoustic communication have

79 been studied extensively across a range of taxa, including birds, marine mammals,

80 amphibians and even invertebrates, there has been limited exploration of these effects in

81 terrestrial mammals (Shannon, McKenna, et al. 2016). Terrestrial mammals display

82 flexibility in call structures relative to social and geophysical conditions (Ey and Fischer

83 2009; Townsend and Manser 2013), and recent work has demonstrated that mongooses

84 exhibit reduced responsiveness to conspecific and heterospecific vocalisations in road

85 noise (Kern and Radford 2016; Morris-drake et al. 2017). However, studies explicitly

86 exploring the effects of noise on vocalisations have largely been limited to research on

87 bats. For example, Brazilian free-tailed bats (Tadarida brasiliensis) reduced the

88 bandwidth of their echolocation search calls when exposed to noise (Bunkley and Barber

89 2015), fringe-lipped bats (Trachops cirrhosus) shifted from targeting prey-generated

90 sources of sound to using echolocation when hunting in noise (Gomes et al. 2016), and

91 Asian particolored bats (Vespertilio sinensis) simplified the complexity and raised the

92 amplitude of their social calls when exposed to traffic noise (Jiang et al. 2019) but did not 
93 adjust the vocal rate or duration of these vocalisations (Song et al. 2019). Other studies

94 have also explored shifts in frequency and amplitude of echolocating bats, but the

95 researchers exposed the animals to noise with a specific frequency (bandpass filtered),

96 compared with the broadband frequencies that are typical of anthropogenic noise (Hage

97 et al. 2013; Hage et al. 2014). The paucity of research on a wider range of mammal

98 species risks overlooking the impacts of a key anthropogenic stressor on terrestrial

99 systems.

100 In this paper, we explore whether a gradient of increasing urban traffic and

101 associated environmental noise affects the alarm call characteristics of black-tailed prairie

102 dogs (Cynomys ludovicianus) - hereafter referred to as prairie dogs. Prairie dogs are prey

103 species for a wide range of grassland predators including badgers (Taxidea taxus),

104 coyotes (Canis latrans), hawks and snakes (Hoogland 1995). Alarm calls - a series of

105 rapid high-pitched barks - provide one of the key anti-predator strategies employed by

106 this group-living species (Hoogland 1995), but the production of these calls appears to be

107 influenced by social context. For example, prairie dogs have been shown to give alarm

108 calls more readily when in the presence of kin compared to unrelated conspecifics

109 (Hoogland 1983; Hoogland 1995). This provides evidence that the seemingly costly

110 behaviour of an individual alerting a predator to their presence may have indirect fitness

111 benefits (Shelley and Blumstein 2005). Moreover, we recently demonstrated that the

112 presence of young influenced the alarm call characteristics of adult prairie dogs -

113 whereby they lowered the central concentration of energy in their calls (Wilson-Henjum

114 et al. 2019). The social context and function of alarm call production provides an

115 interesting avenue for exploring the effects of exposure to anthropogenic noise on animal 
116 vocalisation, particularly when contrasted with findings from the significant body of

117 work focussing on advertisement calls and songs (reviewed by Shannon, McKenna, et al. 118 2016).

119 Although prairie dog populations across the United States have been dramatically 120 reduced as a result of land-use changes and disease (Miller, Ceballos, \& Reading, 1994;

121 Miller et al., 2007), they have shown the ability to inhabit urban environments (Magle et

122 al. 2010; Magle and Fidino 2018). In common with other wildlife species that can survive

123 in human-dominated landscapes, this persistence is likely to be a function of their

124 behavioural flexibility, which allows them to adjust to the environmental conditions of

125 their surroundings (Lowry et al. 2013). Prairie dogs therefore provide an interesting study

126 species for furthering our understanding of behavioural and demographic responses to

127 anthropogenic disturbance in a social mammal. In addition to exploring vocal plasticity

128 relative to noise exposure in a free-ranging terrestrial mammal, this study also focuses on

129 a form of vocal communication that has received less attention in this field of research -

130 alarm calling to signal the presence of a perceived threat (Potvin et al. 2014; Templeton

131 et al. 2016). While calls and songs aimed at attracting mates and defending territories

132 play a crucial role in the reproductive success of an animal, alarm calls arguably have an

133 even more immediate and profound effect on fitness through the mediation of survival.

134 Our previous research found that prairie dogs exposed to noise adjusted their

135 vigilance and foraging behaviour, consistent with the risk disturbance hypothesis, which

136 predicts anthropogenic disturbance will elicit increased antipredator behaviour (Shannon

137 et al. 2014). Because of their enhanced vigilance, prairie dogs detected and responded to

138 an approaching predator quicker in noise than during the ambient control - contrary to the 
139 distracted prey hypothesis (Shannon, et al., 2016). Here, we explore whether prairie dogs

140 exhibit vocal plasticity in noise - a potential mechanism to overcome acoustical masking

141 - to further illustrate how prairie dogs perceive and respond to this novel pollutant. This

142 will not only broaden the types of communication studied in the context of increasing

143 anthropogenic noise, but has implications for conserving animals in evolutionarily novel

144 environments, such as urban areas that are dramatically expanding with human

145 population growth. We predicted that prairie dogs would elevate the lower frequency

146 limit of their alarm calls when exposed to increasing road traffic noise - so as to

147 minimize acoustical masking.

\section{Methods}

150 Study sites

151 The study was conducted across three prairie dog colonies in predominantly shortgrass

152 prairie habitat located within or adjacent to the city of Fort Collins, Colorado, USA. The

153 sites were selected to provide a gradient of exposure to urban traffic and associated noise.

154 Pineridge Natural Area (250 ha), located on the western edge of the city with a small

155 country road on the northwest boundary ( $\sim 750 \mathrm{~m}$ from the center of the colony),

156 experiences the least anthropogenic noise of the three colonies and is a site that we have

157 used for previous research on prairie dog responses to road traffic noise (Shannon et al.

158 2016). Coyote Ridge Natural Area, situated close to the southwest boundary of the city, is

159840 ha in extent and adjacent to a larger open space to the south and west; the center of

160 the prairie dog colony is located $\sim 350 \mathrm{~m}$ from the relatively busy County Road 19 . The

161 Coterie Natural Area is a small (1.6 ha) site located within the city at the intersection of 
162 two main roads ( $\sim 50 \mathrm{~m}$ to the center of the colony), resulting in considerable levels of

163 urban noise. All three of the sites can be accessed by trails that are used by walkers,

164 runners and cyclists. The prairie dogs are therefore regularly exposed to human activity.

166 Alarm call measurements

167 Prairie dog alarm calls were recorded from 28 August to 6 December 2014 using a Rode

168 NTG-2 shotgun microphone, which was connected to a Roland Moore R-05 digital

169 recorder. Data collection was carried out during daylight hours $(0700-1900)$ by the

170 same single observer $(\mathrm{GWH})$. Alarm calls were elicited by the observer approaching a

171 randomly selected prairie dog - with a systematic approach employed to ensure that

172 different areas of the colony (and animals) were sampled from one study site visit to the

173 next. Once the prairie dog began alarm calling the observer remained stationary and

174 recorded 30 seconds of vocalization while the animal was in situ. Distance to the target

175 animal and the distance from this individual to their nearest neighbor was measured using

176 a laser range finder. All calls were recorded within a distance of $18 \mathrm{~m}$ from the animal

177 (mean $\pm \mathrm{SD}=9 \mathrm{~m} \pm 3$ ) with small differences between sites (Pineridge $=10 \mathrm{~m} \pm 2$, Coyote

178 Ridge $=11 \mathrm{~m} \pm 3$, The Coterie $=8 \mathrm{~m} \pm 2$ ). In order to reduce the possibility that the same

179 prairie dog was selected more than once during the same recording session, the observer

180 ensured that there was a minimum of $30 \mathrm{~m}$ (the average size of a burrow system; Sheets et

181 al. 1971) between the individuals targeted for inclusion in the study. Wind speed and the

182 prevailing weather conditions were all documented at the time of recording. A total of

183137 alarm call recording periods were collected across the three sites (Pineridge $=46$,

184 Coyote Ridge $=44$, The Coterie $=47$ ). 
A band-limited automated detector was used in Raven Pro v1.5 to select each of

186 the individual barks in the 30 -second calling bouts and to optimize extraction of call

187 parameters. The following settings were used in the detector: minimum frequency of

$1882000 \mathrm{~Hz}$, maximum frequency of $15000 \mathrm{~Hz}$, minimum signal duration of 0.008 seconds,

189 maximum signal duration of 0.2 seconds, minimum separation of 0.2 seconds, minimum

190 occupancy of 30 percent, and a signal-to-noise threshold of $15 \mathrm{~dB}$. Before measurements

191 were extracted on the individual barks, all detections were examined manually for

192 accuracy and adjusted to maximize the detection of all barks within a recording period

193 and to ensure the entire bandwidth and duration of calls were selected. Because prairie

194 dogs produce short duration, broadband barks, a standardized maximum frequency

$195(15000 \mathrm{~Hz})$ was used for each detection box. Random selections of half of the barks in a 196 calling bout $(\mathrm{n}=4516)$ were then measured.

197 Four acoustic metrics were calculated for each bark: (1) minimum frequency (Hz)

198 - the lower frequency limit of the call, a commonly used metric in previous studies; (2)

199 frequency $5 \%(\mathrm{~Hz})$ - the frequency where the summed energy equals $5 \%$ of the total, a

200 measure of lower frequency properties; (3) peak frequency $(\mathrm{Hz})$ - the frequency with the

201 highest concentration of energy; and (4) bark duration (milliseconds) (Figure 1).

203 Ambient sound level measurements

204 Ambient sound levels were measured using a calibrated Larson-Davis 831 sound

205 level meter (frequency weighting $=$ A) over a 2-minute period as soon as the vocalization

206 recording was completed. Sound pressure levels were measured as 1-second frequency

207 weighted $(12.5 \mathrm{~Hz}-20 \mathrm{kHz})$ equivalent continuous levels (LAeq, 1s). Although ambient 
208 sound levels may fluctuate slightly from the time that the alarm call was recorded to the

209 time that the sound pressure level was measured, we believe this variation was minimal

210 relative to overall variation in ambient sound levels across sampling events and sites.

211 Furthermore, it was not possible to conduct the measurements simultaneously, as the

212 ambient sound level recordings would have been biased from the alarm call of the prairie

213 dog. The sound pressure levels were downloaded with the SLM Utility-G3 and

214 customized scripts in $\mathrm{R}$ were used to calculate the $\mathrm{L}_{\text {Aeq }}$ over 120 seconds associated with

215 each recording period (see Electronic Supplementary Material for details).

\section{Statistical analysis}

218 To explore differences in prairie dog vocalisations across the three colonies, alarm call

219 characteristics were initially analysed using a one-way ANOVA with Tukey's HSD.

220 Response variables included the four acoustic metrics described above, and the analysis 221 calculated the mean call characteristics for each target animal, averaged across multiple

222 barks within a bout of alarm calling. The distribution of the residuals was plotted to

223 check that the assumptions of the model were met (e.g., normality and homogeneity). To

224 reduce the likelihood of type 1 errors with multiple comparisons of call parameters across

225 sites, we used an alpha level of 0.01 to assess statistical significance.

226 Next, a generalized linear mixed model (GLMM) framework using the lme4

227 package in $\mathrm{R}$ ( $\mathrm{R}$ Core Development Team 2019) was used to understand the conditions 228 that correlate with changes in alarm call characteristics. Response variables included the

229 four-acoustic metrics, and characteristics of each individual bark were entered into the 230 analyses with the individual observation number included as a random effect to account 
231 for the repeated measures (multiple barks) within a given alarm call. Akaike's

232 Information Criterion adjusted for small sample size (AICc) was used for model selection

233 (Burnham and Anderson 2002). A total of 29 candidate models were generated for each

234 of the response variables using combinations of five predictor variables (Table 1).

235 Predictor variables included the ambient sound level $\left(L_{A e q, 120 s}\right)$ when the calls bouts were

236 recorded, Julian day to establish if there was a change in response over the course of the

237 fieldwork, distance recorded to account for variation in the distance between the observer

238 and the target animal, wind speed to control for the influence of fluctuating acoustic

239 conditions, and distance to the nearest neighbor to determine if proximity to a

240 conspecific influences the observed alarm call response (Table 1). Two interactions were

241 also included to determine whether the effect of noise level exposure on acoustic

242 parameters was modulated by distance to the observer (ambient sound level * distance

243 recorded), and/or distance to the nearest prairie dog (ambient sound level * distance to

244 the nearest neighbor). These predictor variables were normalized so that the relative

245 contribution could be determined in the model averaged output (Table 2). The

246 AICcmodavg package was used to extract AICc scores and model weights for candidate

247 models of each response variable. Model averaging was conducted across models

248 accounting for $\geq 0.95$ of the AICc weight to extract parameter $\beta$ estimates and their $95 \%$

249 confidence intervals (CI). The significance of the results was assessed by whether the

$25095 \%$ CI overlapped zero. This research was approved according to Colorado State

251 University Animal Care and Use Committee protocol 13-4112A. 


\section{Results}

255 Pineridge Natural Area was the quietest of the three colonies with ambient sound levels

256 of $26-50 \mathrm{~dB} \mathrm{~L}_{\text {Aeq, } 120 \mathrm{~s}}(\mathrm{~N}=46)$, mean $=36 \mathrm{~dB} \pm 2(95 \% \mathrm{CI})$, while Coyote Ridge

257 experienced ambient sound levels of $34-54 \mathrm{~dB}_{\mathrm{A}}{ }_{\mathrm{Aeq}, 120 \mathrm{~s}}(\mathrm{~N}=45)$, mean $=42 \mathrm{~dB} \pm 1(95 \%$

$258 \mathrm{CI})$ and The Coterie had the highest ambient sound levels $49-76 \mathrm{~dB} \mathrm{~L}_{\text {Aeq,120s }}(\mathrm{N}=47)$,

259 mean $=58 \mathrm{~dB} \pm 2(95 \% \mathrm{CI})$.

260 The minimum frequency of prairie dog alarm calls differed across the three

261 colonies (ANOVA: $F_{2,134}=8.703, P=0.0003$ ); Pineridge had the lowest minimum

262 frequency $($ mean $=1151 \mathrm{~Hz} \pm 197 \mathrm{SD})$ followed by Coyote Ridge $(1218 \mathrm{~Hz} \pm 149 \mathrm{SD})$

263 and The Coterie (1297 Hz $\pm 161 \mathrm{SD}$ : Figure 2a). The Tukey HSD test revealed a

264 significant difference in minimum frequency between Pineridge and The Coterie $(P=$

265 0.0002), but not between Coyote Ridge and The Coterie $(P=0.04)$ and Pineridge and

266 Coyote Ridge $(P=0.22)$. We did not detect significant differences across colonies for

267 frequency 5\% (ANOVA: $\left.F_{2,134}=1.694, P=0.188\right)$, peak frequency (ANOVA: $F_{2,134}=$

$2681.442, P=0.24$ ) or bark duration (ANOVA: $F_{2,134}=1.648, P=0.196$; Figure 2).

269 Minimum frequency of alarm calls was predicted by six top models, with three

270 models contributing $63 \%$ of the AICc weight (Table 2$)$. Ambient sound level $\left(L_{A e q, 120 s}\right)$

271 was a key parameter across these models, with increasing noise predicting elevated

272 minimum frequency of alarm calls (Table 3). None of the other explanatory variables

273 demonstrated a significant relationship with the minimum frequency of alarm calls (Table

$2743)$.

275 The frequency $5 \%$ call property was predicted by 10 top models, with three 276 accounting for $54 \%$ of the AICc weight (Table 2$)$. Ambient sound level $\left(L_{\text {Aeq, } 120 s}\right)$ was not 
277 a significant predictor, with little evidence that higher noise led to a lower frequency

278 alarm call (measured as frequency 5\%) (Table 3). Frequency 5\% was greater for alarm

279 calls recorded at distances further from the observer (Dist recorded) and when calling

280 prairie dogs were closer to the nearest neighbour (Dist Neighbor) (Table 3).

281 Peak frequency was predicted by 11 top models, of which three accounted for

$28250 \%$ of the AICc weight (Table 2). As with the analysis of frequency $5 \%$, there was no

283 evidence of a clear relationship between the ambient sound level and the peak frequency

284 of the call (Table 3). Furthermore, no other variables were significant predictors of peak

285 frequency.

286 Bark duration was predicted by 7 top models, with three contributing $61 \%$ of the

287 AICc weight (Table 2). No explanatory variables had a significant relationship with bark 288 duration (Table 3).

290 Discussion

291 Alarm calls provide crucial information on the presence and proximity of predatory

292 threats - essential for prairie dogs, which are social prey species that are targeted by a

293 number of terrestrial and aerial predators (Hoogland 1995). As such, and in line with

294 previous research on a range of bird and marine mammal species (Shannon, McKenna, et

295 al. 2016), we predicted that prairie dogs would reduce the masking effect of urban noise

296 by increasing the lower frequency limit of their alarm calls when exposed to elevated

297 anthropogenic noise. The evidence for this, however, was limited. We did detect an

298 increase in the minimum frequency of alarm calls with increasing urban noise, but there

299 was no effect of urban noise on the frequency $5 \%$ metric, which is a more robust measure 
300 of the minimum frequency of animal vocalisations (Brumm et al. 2017). Likewise, peak

301 frequency and bark duration of alarm calls were not related to ambient sound levels.

302 Previous studies have also shown that the frequency of bird vocalisations are not

303 consistently adjusted in urban noise across species (Hu and Cardoso 2010), and even

304 when they are modified, they can shift in the opposite direction to that predicted, i.e. with

305 lower frequency calls in noisier conditions potentially to increase transmission distance

306 (Potvin et al. 2014). For taxa with particularly low frequency calls, it may prove too

307 energetically costly (or physiologically challenging) to actually shift the frequency of

308 vocalisation high enough to reduce the risk of masking, while those that use higher

309 frequency calls are less affected by noise and therefore might not need to adjust their calls

310 (Hu and Cardoso 2010). Prairie dogs have short duration alarm calls that extend across a

311 broad range of frequencies from $1 \mathrm{kHz}$ to $>8 \mathrm{kHz}$, with a peak frequency of

312 approximately $3.5 \mathrm{kHz}$, while the energy in urban noise is generally focussed below 2.5

$313 \mathrm{kHz}$. It may well be the case that the relatively high frequency of their vocalisations

314 means that prairie dogs do not experience significant masking from exposure to urban

315 noise.

316 While our findings of an increase in minimum frequency with rising noise level

317 concur with previous studies, scientists have recently questioned the methods used to

318 measure minimum frequency because they may result in false positives (Ríos-Chelén et

319 al. 2017; Brumm et al. 2017). Indeed, the majority of studies on this topic have relied on

320 researchers visually inspecting the spectrogram to determine the minimum vocal

321 frequency, a method that has been shown to potentially bias the results, particularly if the

322 observer has a priori expectations (Ríos-Chelén et al. 2017; Brumm et al. 2017). 
323 Furthermore, the signal to noise ratio in acoustic data can result in the minimum

324 frequency being masked under elevated noise levels, resulting in artificial inflation of the

325 observed minimum frequency (Brumm et al. 2017). It was interesting to note the marked

326 difference in our model results for the minimum frequency and frequency $5 \%$ metrics,

327 further highlighting the risk of using the absolute minimum frequency when exploring

328 vocal adjustments by animals in anthropogenic noise.

329 We found evidence for changes in vocal behaviour related to the social context of the

330 alarm calls. Prairie dogs that were at a greater distance from conspecifics (i.e. more

331 isolated), and therefore may have been at a higher risk of predation, produced calls with

332 lower frequencies. We suggest that this could be a result of reduced call amplitude, which

333 is typically positively correlated with call frequency (Brumm and Naguib 2009; Zollinger

334 et al. 2012; Nemeth et al. 2013) - however it is important to note that we were unable to

335 measure alarm call amplitude in this study. Such a strategy of producing softer low-

336 amplitude calls, documented across a range of species, can reduce eavesdropping and

337 detection by a third-party (Reichard and Anderson 2015), in this case an approaching

338 predator in the form of a human observer. Prairie dogs also produced alarm calls with

339 increased lower frequencies when the observer (i.e., predator threat) was further from the

340 calling animal. Prairie dogs may elevate call amplitude, and consequently generate higher

341 frequency calls, when predators are at a greater distance to increase the likelihood the

342 vocalisation is received across a greater area of the colony, without unduly increasing the

343 risk to the caller. This is especially pertinent given that the function of the alarm call is to

344 both warn conspecifics of approaching danger and to communicate to the predator that

345 they have been detected (Isbell and Bidner 2016). Additional experiments conducted by 
346 our research group demonstrated that prairie dogs adjusted their alarm calls - reducing

347 the central concentration of energy - when calling in the presence of vulnerable pups

348 (Wilson-Henjum et al. 2019). Adjustment in prairie dog communication, therefore,

349 appears to be structured by social context mediated by spatial proximity to an

350 approaching threat. However, this is an area of research that warrants further detailed

351 investigation to reveal the specific drivers of vocal modulation.

352 Unlike many previous studies that have explored the effects of anthropogenic

353 noise on communication, our research focussed on alarm calls rather than songs or

354 vocalisations that animals use to advertise their quality or fitness to conspecifics

355 (reviewed in Shannon, McKenna, et al. 2016). The effective communication distance for

356 an alarm call in a colonial species may be significantly less than that of a call or song

357 aimed at attracting a mate or defending a territory. Therefore, even though the ambient

358 noise levels were considerable (mean of $58 \mathrm{~dB}$ at the Coterie, which is comparable to

359 normal conversation at $1 \mathrm{~m}$ ), they may not be loud enough to sufficiently mask the alarm

360 call from being perceived by nearby conspecifics. This raises a number of interesting

361 future research avenues regarding the function of a given vocalisation and its

362 susceptibility to masking from anthropogenic noise, as well as the plasticity in response

363 exhibited across taxa. It is also important to note that practical limitations meant that we

364 only had three sites in our study design, each with a different noise exposure resulting in

365 some level of pseudoreplication. Ideally, further research on this topic will identify

366 multiple sites at each broad level of noise exposure.

367 Elucidating the specific mechanisms (e.g., distraction, masking, predatory threat,

368 social context) driving behavioural responses to anthropogenic noise can prove 
369 challenging, particularly as they are not necessarily mutually exclusive. Nevertheless, a

370 combination of natural experiments and playback approaches can be used to identify the

371 key mechanisms for specific taxa, which can greatly inform our understanding of the

372 effects of noise, as well as assist in developing effective mitigation of these impacts

373 (Francis and Barber 2013). Our work on free-ranging prairie dogs has demonstrated that

374 they adjust critical behaviours when exposed to noise - including increased vigilance and

375 reduced foraging - which suggests that noise is responded to as an elevated level of

376 perceived risk (Shannon et al. 2014). Furthermore, in contrast to a number of aquatic

377 species (Chan et al. 2010; Wale et al. 2013; Simpson et al. 2015), prairie dogs did not

378 exhibit distraction from an approaching predator under noisy conditions - indeed, they

379 actually became alert and took flight sooner in traffic noise than under quieter control

380 conditions (Shannon et al. 2016). While the findings presented here suggest that the

381 acoustic characteristics of prairie dog alarm calls are consistent across a broad range of

382 ambient noise levels, indicating that masking may not be a key driver shaping their vocal

383 behaviour under these conditions.

\section{Funding}

386 The National Park Service Natural Sounds and Night Skies Division funded this research.

388 Acknowledgements

389 We would like to thank J. Shanahan and A. Meyer at Fort Collins Natural Areas for

390 logistical support and permission to conduct the study, and L. Cordes for statistical

391 advice. 
Data accessibility: Analyses reported in this article can be reproduced using the data

393 provided by Shannon et al. (2019).

\section{References}

Barber JR, Crooks KR, Fristrup KM. 2010. The costs of chronic noise exposure for terrestrial organisms. Trends Ecol. Evol. 25:180-189.

Brumm H, Naguib M. 2009. Environmental Acoustics and the Evolution of Bird Song. In: M Naguib, K, Zuberbuhler, NS Clayton VJ, editor. Advances in the Study of Behavior. Vol. 40. Academic Press. p. 1-33.

Brumm H, Zollinger SA, Niemelä PT, Sprau P. 2017. Measurement artefacts lead to false positives in the study of birdsong in noise. Methods Ecol. Evol. 11:1617-1625.

\section{Bunkley JP, Barber JR. 2015. Noise Reduces Foraging Efficiency in Pallid Bats} (Antrozous pallidus). Ethology 121:1116-1121.

Burnham K, Anderson D. 2002. Model selection and multimodel inference: a practical information-theoretic approach. Springer Science \& Business Media.

Chan AAY-H, Giraldo-Perez P, Smith S, Blumstein DT. 2010. Anthropogenic noise affects risk assessment and attention: the distracted prey hypothesis. Biol. Lett. $6: 458-461$.

Díaz M, Parra A, Gallardo C. 2011. Serins respond to anthropogenic noise by increasing vocal activity. Behav. Ecol. 22:332-336.

Ey E, Fischer J. 2009. The "Acoustic adaptation hypothesis" - A review of the evidence from birds, anurans and mammals. Bioacoustics 19:21-48.

Francis CD. 2015. Vocal traits and diet explain avian sensitivities to anthropogenic noise. Glob. Chang. Biol. 21:1809-1820.

Francis CD, Barber JR. 2013. A framework for understanding noise impacts on wildlife: an urgent conservation priority. Front. Ecol. Environ. 11:305-313.

Francis CD, Ortega CP, Cruz A. 2009. Noise Pollution Changes Avian Communities and Species Interactions. Curr. Biol. 19:1415-1419.

Fuller RA, Warren PH, Gaston KJ. 2007. Daytime noise predicts nocturnal singing in 
urban robins. Biol. Lett. 3:368-70.

423 Gomes DGE, Page RA, Geipel I, Taylor RC, Ryan MJ, Halfwerk W. 2016. Bats perceptually weight prey cues across sensory systems when hunting in noise. Science 353:1277-1280.

Hage S, Jiang T, Berquist S, Feng J, Metzner W. 2013. Ambient noise induces independent shifts in call frequency and amplitude within the Lombard effect in echolocating bats. Proc. Natl. Acad. Sci. 110:4063-4068.

Hage SR, Jiang T, Berquist SW, Feng J, Metzner W. 2014. Ambient noise causes independent changes in distinct spectro-temporal features of echolocation calls in horseshoe bats. J. Exp. Biol. 217:2440-4.

Hoogland JL. 1983. Nepotism and alarm calling in the black-tailed prairie dog (Cynomys ludovicianus). Anim. Behav. 31:472-479.

Hoogland JL. 1995. The Black-Tailed Prairie Dog: Social Life of a Burrowing Mammal. University of Chicago Press.

$\mathrm{Hu}$ Y, Cardoso GC. 2010. Which birds adjust the frequency of vocalizations in urban noise? Anim. Behav. 79:863-867.

Isbell LA, Bidner LR. 2016. Vervet monkey (Chlorocebus pygerythrus) alarm calls to leopards (Panthera pardus) function as a predator deterrent. Behaviour 153:591606.

Jiang T, Guo X, Lin A, Wu H, Sun C, Feng J, Kanwal JS. 2019. Bats increase vocal amplitude and decrease vocal complexity to mitigate noise interference during social communication. Anim. Cogn. 22:199-212.

Kern JM, Radford AN. 2016. Anthropogenic noise disrupts use of vocal information about predation risk. Environ. Pollut. 218:988-995.

Lowry H, Lill A, Wong BBM. 2012. How Noisy Does a Noisy Miner Have to Be? Amplitude Adjustments of Alarm Calls in an Avian Urban 'Adapter.' PLoS One 7:e29960.

Lowry H, Lill A, Wong BBM. 2013. Behavioural responses of wildlife to urban environments. Biol. Rev. 88:537-549.

Magle SB, Fidino M. 2018. Long-term declines of a highly interactive urban species. Biodivers. Conserv. 27:3693-3706. 
Magle SB, Reyes P, Zhu J, Crooks KR. 2010. Extirpation, colonization, and habitat dynamics of a keystone species along an urban gradient. Biol. Conserv. 143:21462155.

Miller B, Ceballos G, Reading R. 1994. The prairie dog and biotic diversity. Conserv. Biol. 8:677-681.

Miller BJ, Reading RP, Biggins DE, Detling JK, Forrest SC, Hoogland JL, Javersak J, Miller SD, Proctor J, Truett J, et al. 2007. Prairie Dogs: An Ecological Review and Current Biopolitics. J. Wildl. Manage. 71:2801-2810.

Morris-drake A, Bracken AM, Kern JM, Radford AN. 2017. Anthropogenic noise alters dwarf mongoose responses to heterospecific alarm calls. Environ. Pollut. 223:476483.

Nemeth E, Pieretti N, Zollinger SA, Geberzahn N, Partecke J, Brumm H, Miranda AC. 2013. Bird song and anthropogenic noise : vocal constraints may explain why birds sing higher-frequency songs in cities. Proc. R. Soc. B Biol. Sci. 280:20122798.

Potvin DA, Mulder RA, Parris KM. 2014. Silvereyes decrease acoustic frequency but increase efficacy of alarm calls in urban noise. Anim. Behav. 98:27-33.

Potvin DA, Parris KM, Mulder RA. 2011. Geographically pervasive effects of urban noise on frequency and syllable rate of songs and calls in silvereyes (Zosterops lateralis). Proc. Biol. Sci. 278:2464-9.

Proppe DS, Sturdy CB, St. Clair CC. 2013. Anthropogenic noise decreases urban songbird diversity and may contribute to homogenization. Glob. Chang. Biol. 19:1075-1084.

R Core Development Team. 2019. R: a language and environment for statistical computing. Vienna (Austria): R Foundation for Statistical Computing.

Reichard DG, Anderson RC. 2015. Why signal softly? The structure, function and evolutionary significance of low-amplitude signals. Anim. Behav. 105:253-265.

Ríos-Chelén AA, McDonald AN, Berger A, Perry AC, Krakauer AH, Patricelli GL. 2017. Do birds vocalize at higher pitch in noise, or is it a matter of measurement? Behav. Ecol. Sociobiol. 71:1-12.

Shannon G, Angeloni LM, Wittemyer G, Fristrup KM, Crooks KR. 2014. Road traffic noise modifies behaviour of a keystone species. Anim. Behav. 94:135-141. 
Shannon G, Crooks KR, Wittemyer G, Fristrup KM, Angeloni LM. 2016. Road noise causes earlier predator detection and flight response in a free-ranging mammal. Behav. Ecol. 27:1370-1375.

Shannon G, McKenna MF, Angeloni LM, Crooks KR, Fristrup KM, Brown E, Warner KA, Nelson MD, White C, Briggs J, et al. 2016. A synthesis of two decades of research documenting the effects of noise on wildlife. Biol. Rev. 91:982-1005.

Shannon G, McKenna MF, Wilson-Henjum GE, Angeloni LM, Crooks KR, Wittemyer G. 2019. Data from: Vocal characteristics of prairie dog alarm calls across an urban noise gradient. Behav. Ecol. doi: https://doi.org/10.5061/dryad.vmcvdncp9

Sheets RG, Linder RL, Dahlgren RB. 1971. American Society of Mammalogists Burrow Systems of Prairie Dogs in South Dakota. Source J. Mammal. 52:451-453.

Shelley EL, Blumstein DT. 2005. The evolution of vocal alarm communication in rodents. Behav. Ecol. 16:169-177.

Sih A, Ferrari MCO, Harris DJ. 2011. Evolution and behavioural responses to humaninduced rapid environmental change. Evol. Appl. 4:367-387.

Simpson SD, Purser J, Radford AN. 2015. Anthropogenic noise compromises antipredator behaviour in European eels. Glob. Chang. Biol. 21:586-593.

Slabbekoorn H. 2013. Songs of the city: noise-dependent spectral plasticity in the acoustic phenotype of urban birds. Anim. Behav. 85:1089-1099.

Slabbekoorn H, Ripmeester EAP. 2008. Birdsong and anthropogenic noise: implications and applications for conservation. Mol. Ecol. 17:72-83.

Song S, Lin A, Jiang T, Zhao X, Metzner W, Lin A, Feng J. 2019. Bats adjust temporal features of echolocation calls but not those of communication calls in response to traffic noise. Integr. Zool.

Templeton CN, Zollinger SA, Brumm H. 2016. Traffic noise drowns out great tit alarm calls. Curr. Biol. 26:R1173-R1174.

Townsend SW, Manser MB. 2013. Functionally Referential Communication in Mammals : The Past, Present and the Future. 119:1-11.

Wale M a., Simpson SD, Radford AN. 2013. Noise negatively affects foraging and antipredator behaviour in shore crabs. Anim. Behav. 86:111-118.

Wilson-Henjum GE, Job JR, McKenna MF, Shannon G, Wittemyer G. 2019. Alarm call 
515 modification by prairie dogs in the presence of juveniles. J. Ethol. 37:167-174.

516 Zollinger SA, Podos J, Nemeth E, Goller F, Brumm H. 2012. On the relationship

517 between, and measurement of, amplitude and frequency in birdsong. Anim. Behav.

$518 \quad 84: \mathrm{e} 1-\mathrm{e} 9$.

519

520

521

522

523

524

525

526

527

528

529

530

531

532

533

534

535

536

537

538

539

540

541

542

543 


\section{$544 \quad$ Figure legends}

545 Figure 1. Spectrogram of black-tailed prairie dog alarm calls collected from the colony at

546 The Coterie Natural Area. The extracted call parameters are shown for a single call.

547 Spectrogram parameters: 512 fast Fourier transformation, Hann window, 50\% overlap,

$54893-\mathrm{Hz}$ frequency resolution, $3.25 \mathrm{~ms}$ temporal resolution. The dark band of energy below

$5492.5 \mathrm{kHz}$ is generated by urban noise at the study site.

550

551 Figure 2. Mean $( \pm 95 \% \mathrm{CI})$ values for the four acoustic metrics extracted from prairie

552 dog calls $(n=137)$ across the three study sites with increasing noise exposure from left to 553 right. 
565 Table 1. Structure of candidate models assessed for the four response variables

566 (minimum frequency, frequency 5\%, peak frequency and bark duration). Individual

567 observation number was included as a random effect.

568

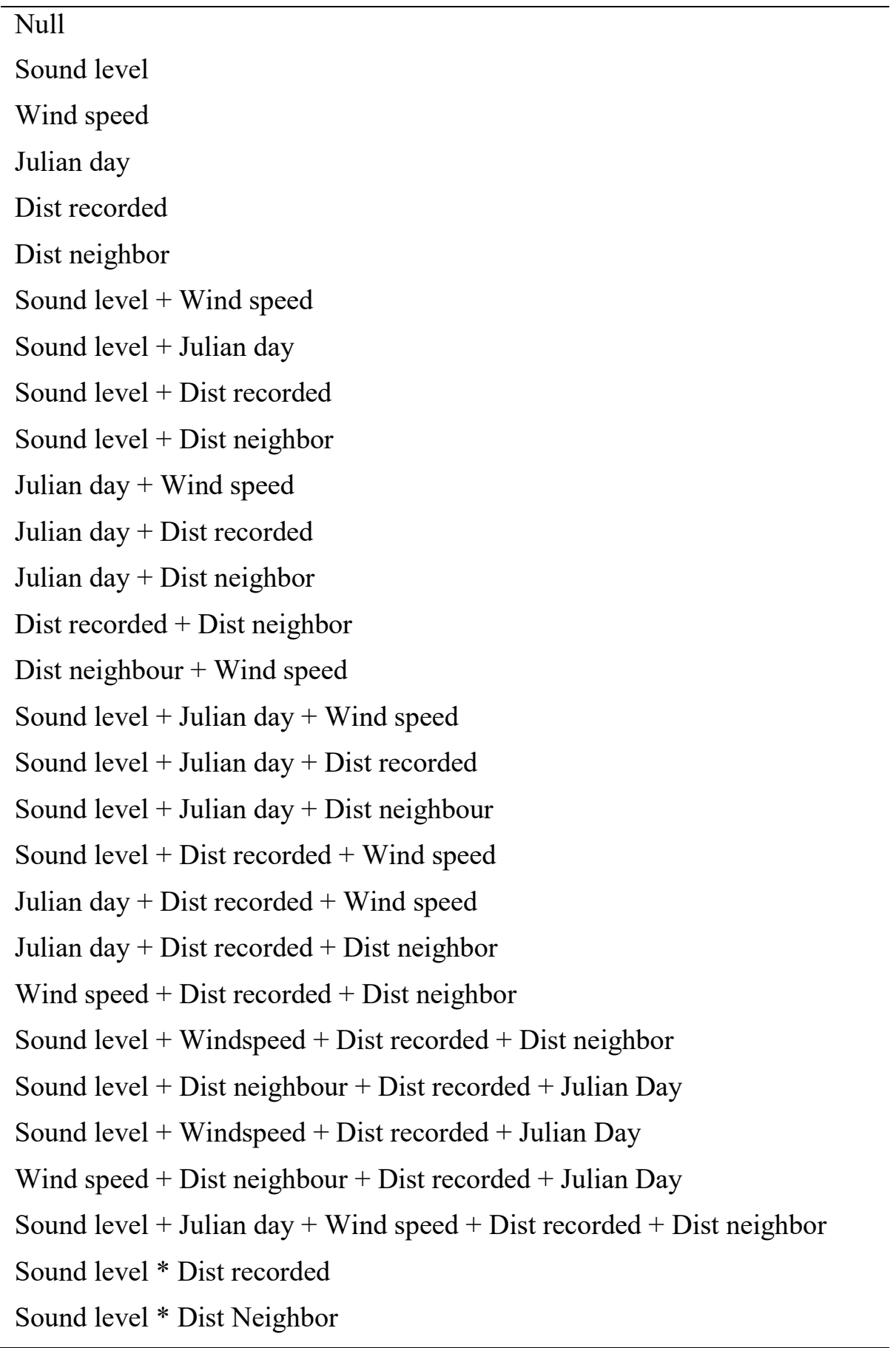


570 Table 2. Top models for the four-acoustic metrics of prairie dog alarm calls $(\geq 0.95$ of the

571 AICc weight). All models include the individual observation number as a random effect.

572

\begin{tabular}{llll}
\hline & K & AICc & AICc w \\
\hline a) Minimum frequency & & & \\
Sound level + Dist recorded + Julian Day + Dist neighbor & 7 & 0.00 & 0.29 \\
Sound level * Dist neighbor & 6 & 0.84 & 0.19 \\
Sound level + Dist neighbor & 5 & 1.24 & 0.1 \\
Sound level + Dist recorded + Dist neighbour + Wind speed & 7 & 1.54 & 0.13 \\
Sound level + Dist recorded + Julian Day + Dist neighbor + Wind speed & 8 & 1.70 & 0.12 \\
Sound level + Julian Day + Dist neighbor & 6 & 1.92 & 0.1
\end{tabular}

\section{a) Frequency 5\%}

Dist recorded + Dist neighbor

Julian Day + Dist recorded + Dist neighbor

Wind speed + Dist recorded + Dist neighbor

Dist recorded + Dist neighbor + Julian Day + Wind speed

Dist neighbor

Sound level + Dist recorded + Julian Day + Dist neighbor

Sound level + Dist recorded + Dist neighbor + Wind speed

Dist neighbor + Wind speed

Sound level + Dist recorded + Julian Day + Dist neighbor + Wind speed

Julian Day + Dist neighbor

50.00

0.25

$6 \quad 1.12$

0.15

$6 \quad 1.12$

0.14

$7 \quad 2.11$

0.09

$4 \quad 2.59$

0.07

$7 \quad 2.78$

0.06

$7 \quad 2.90$

0.06

$5 \quad 3.48$

0.05

$8 \quad 3.71$

0.04

$5 \quad 3.81$

0.04

\section{b) Peak frequency}

Dist neighbour

$4 \quad 0.00$

0.19

Dist recorded * Dist neighbor

$\begin{array}{ll}6 & 0.27\end{array}$

0.16

Dist recorded + Dist neighbor

$5 \quad 0.49$

0.15

Dist neighbor + Wind speed

$5 \quad 1.12$

0.11

Sound level + Dist neighbor

$5 \quad 1.71$

0.08

Wind speed + Dist recorded + Dist neighbor

$6 \quad 1.75$

0.08

Julian day + Dist neighbor

$5 \quad 2.00$

0.07

Julian day + Dist recorded + Dist neighbor

$6 \quad 2.50$

0.05

Sound level + Julian day + Dist neighbor

$6 \quad 3.70$

0.03

Wind speed + Dist neighbor + Dist recorded + Julian Day

$7 \quad 3.76$

0.03

Sound level + Windspeed + Dist recorded + Dist neighbor

$7 \quad 3.76$

0.03 
c) Bark duration

Dist neighbor

Julian day + Dist neighbor

Sound level + Dist neighbor

Dist recorded + Dist neighbor

Sound level + Julian Day + Dist neighbor

Sound level * Dist neighbor

Julian day + Dist recorded + Dist neighbor

$\begin{array}{lll}4 & 0.00 \quad 0.28\end{array}$

$\begin{array}{lll}5 & 1.03 & 0.17\end{array}$

$\begin{array}{lll}5 & 1.04 & 0.16\end{array}$

$\begin{array}{lll}5 & 1.75 & 0.12\end{array}$

$\begin{array}{lll}6 & 2.27 & 0.09\end{array}$

$\begin{array}{lll}6 & 2.32 & 0.09\end{array}$

$\begin{array}{lll}6 & 2.76 & 0.07\end{array}$

573

574

575

576

577

578

579

580

581

582

583

584

585

586

587

588 
589 Table 3. The observed relationship between each response variable and the model-

590 averaged parameters from the top models ( $\beta$-estimate $\pm 95 \% \mathrm{CI}$ ). Bold text denotes $\beta$ -

591 estimates with $95 \% \mathrm{CI}$ that do not overlap zero.

592

\begin{tabular}{|c|c|c|c|}
\hline & Parameter & $\beta$ Estimate & $(95 \% \mathrm{CI})$ \\
\hline \multirow[t]{6}{*}{ Minimum frequency } & Sound level & 67.63 & $(20.18 / 115.09)$ \\
\hline & Dist recorded & 44.16 & $(-0.27 / 88.58)$ \\
\hline & Dist neighbor & 8.10 & $(-33.65 / 49.85)$ \\
\hline & Julian day & 25.29 & $(-13.03 / 63.60)$ \\
\hline & Wind speed & -1.27 & $(-6.26 / 3.72)$ \\
\hline & Sound level * Dist neighbor & -40.89 & $(-92.22 / 10.44)$ \\
\hline \multirow[t]{5}{*}{ Frequency $5 \%$} & Sound level & 17.02 & $(-100.00 / 134.04)$ \\
\hline & Dist recorded & 125.31 & $(11.3 / 239.31)$ \\
\hline & Dist neighbor & -144.57 & $(-258.96 /-30.18)$ \\
\hline & Julian day & 52.54 & $(-52.68 / 157.76)$ \\
\hline & Wind speed & -6.93 & $(-20.72 / 6.86)$ \\
\hline \multirow[t]{6}{*}{ Peak frequency } & Sound level & -50.22 & $(-176.87 / 76.43)$ \\
\hline & Dist recorded & 69.38 & $(-44.69 / 183.46)$ \\
\hline & Dist neighbor & -45.05 & $(-159.71 / 69.61)$ \\
\hline & Julian day & -2.74 & $(-109.24 / 103.77)$ \\
\hline & Wind speed & -6.38 & $(-20.29 / 7.53)$ \\
\hline & Sound level * Dist neighbor & -134.37 & $(-274.70 / 5.96)$ \\
\hline \multirow[t]{6}{*}{ Bark duration } & Sound level & 0.91 & $(-1.39 / 3.22)$ \\
\hline & Dist recorded & -0.55 & $(-3.08 / 1.98)$ \\
\hline & Dist neighbor & 0.12 & $(-2.37 / 2.61)$ \\
\hline & Julian day & -1.13 & $(-3.44 / 1.18)$ \\
\hline & Wind speed & -0.02 & $(-0.33 / 0.28)$ \\
\hline & Sound level $*$ Dis neighbor & -1.34 & $(-4.43 / 1.74)$ \\
\hline
\end{tabular}

593

594 